Document downloaded from:

http://hdl.handle.net/10251/132998

This paper must be cited as:

Pace-Bedetti, HM.; Dolz, JF.; Martínez-De-Juan, JL.; Conejero Rodilla, A. (2019). The effect of postural freedom to increase the neutral positions during laparoscopic surgery. International Journal on Interactive Design and Manufacturing (IJIDeM). 13(2):627-631. https://doi.org/10.1007/s12008-018-00527-6

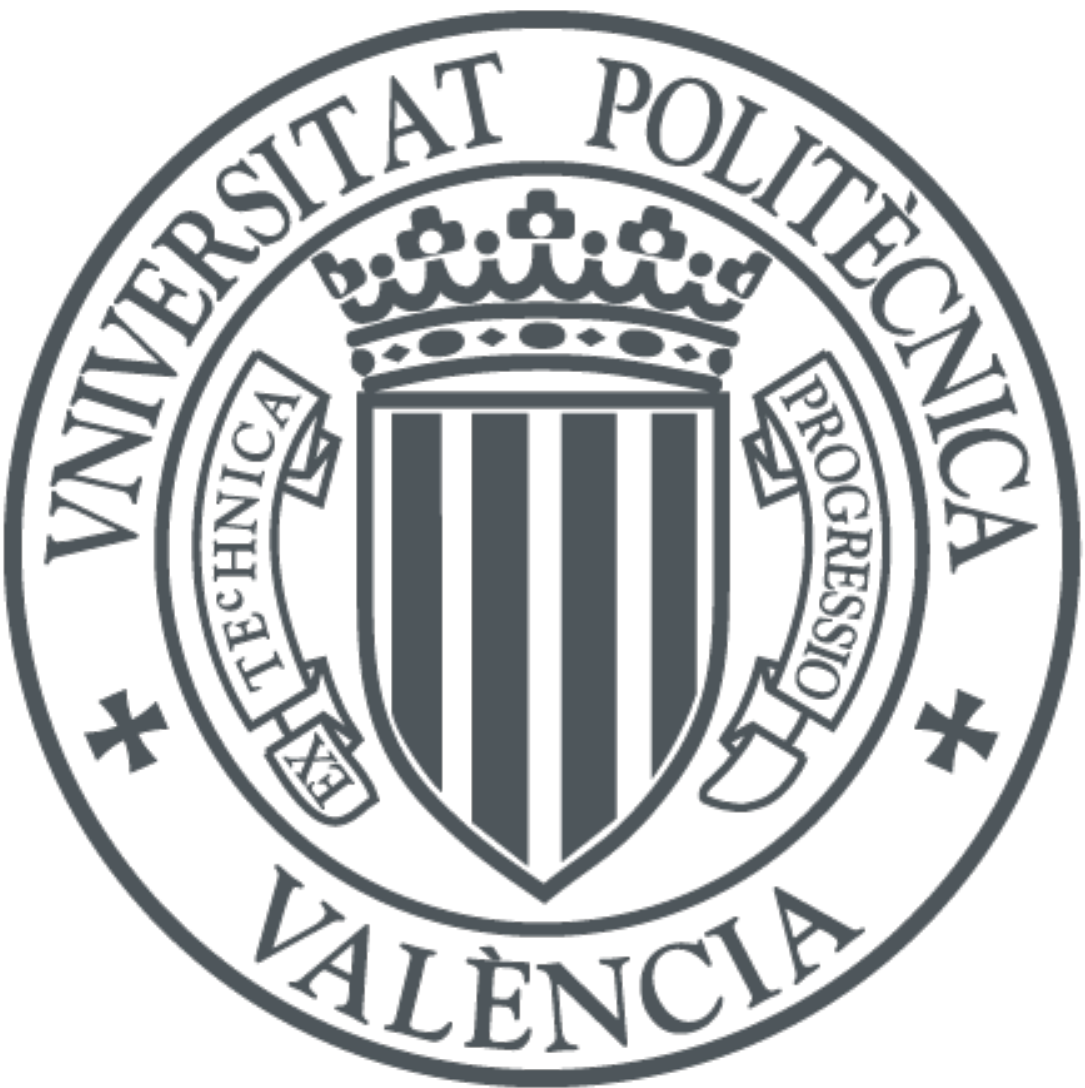

The final publication is available at

https://doi.org/10.1007/s12008-018-00527-6

Copyright Springer-Verlag

Additional Information 


\section{The effect of postural freedom to increase the neutral positions during laparoscopic surgery}

Horacio M. Pace-Bedetti ${ }^{1}$

Instituto de Diseño y Fabricación (IDF), Universidad Politécnica de Valencia

Camí de Vera, s/n, 46022, Valencia, Spain

horaciopacebedetti@gmail.com

+1 (857) 250-1267

José F Dolz

Hospital la Fe de Valencia

Avinguda de Fernando Abril Martorell, 106, 46026, Valencia, Spain

\section{José L. Martínez-de-Juan}

Centro de Investigación e Innovación en Bioingeniería (CI2B), Universidad Politécnica de Valencia

Camí de Vera, s/n, 46022, Valencia, Spain

\section{Andrés Conejero}

Instituto de Diseño y Fabricación (IDF), Universidad Politécnica de Valencia Camí de Vera, s/n, 46022, Valencia, Spain

\section{ACKNOWLEDGMENT}

The authors are grateful to all surgeons and residents who participated in the study and all the team of the clinical simulation area of the "Hospital La Fe" for making this study possible.

${ }_{1}^{1}$ Corresponding author: Horacio M. Pace Bedetti 


\author{
Abstract \\ Laparoscopic technique has demonstrated numerous advantages compared to \\ open conventional surgery. Nevertheless, this procedure increases the surgeons \\ fatigue and thus, the potential to commit errors that may harm the patient during \\ the operation. The post-surgery pain is also augmented because the surgeons \\ are forced to adopt non-neutral postures during the practice. This study reveals \\ how a Postural Freedom element could help surgeons to improve the postural \\ hygiene. \\ During this study, thirteen participants with and without previous \\ experience in laparoscopic surgery performed a test with two instruments: a \\ prototype that implement this postural freedom concept and a conventional fixed \\ instrument.
}

The results obtained indicate that the postural freedom element allows the participants to maintain neutral positions during greatest part of the experiment and suggest that the implementation of an articulated element could increases the neutral positions adopted during a real laparoscopic procedure. The use of the postural freedom concept allowed to the participants to reduce the awkward positions during upper limb motions and to reduce displacements, avoiding extreme abductions that are common with the conventional fixed instruments.

\title{
Keywords
}

Motion analysis; laparoscopic surgery; ergonomics; medical devices. 
Minimal Access Surgery (MAS), also called Minimal Invasive Surgery (MIS), has been one of the main surgical advances in the last few decades. Studies had shown the advantages of minimal access procedures compared to open conventional ones. The reduction in surgical invasiveness results in reductions of procedure time, post-operation pain, trauma, blood-loss, recovery time and risk of infection [1-6].

Despite all this benefits, during MAS, the surgeons are forced to do some concessions. These procedures involve more effort, concentration and greater mental stress [3, 7]. Surgeons are forced to adopt awkward postures and exert substantially higher muscle force on fingers, hands, wrist and arms [8-11]. Fatiguing the surgeons body and creating potential for errors that may harm the patient during the operation [12].

Awkward postures also affect surgeons after operations. In fact, one of the leading causes of surgeon post-operation pain or numbness is the non-neutral postures adopted during laparoscopy [13]. A poor ergonomic posture accelerates the muscle fatigue and pain process because, outside the neutral range, muscles require more energy to generate the same contractile force than in neutral positions [14]. Previous authors tried to solve the complications of these procedures increasing the degrees of freedom (DoF) in the end effector $[2,4,9]$ obtaining positive results but not completely solving the scarcity of neutral postures in wrist, arm and forearm. 


\section{Materials and methods}

Thirteen surgeons and residents of the Hospital La Fe in Valencia (Spain) have been tested. Both women and men of all ages and sizes were included in the study without discrimination. The participants performed the study with their dominant arm. Seven of these participants has no previous experience with this practice (novice). Was consider as an expert, a participant that could be influenced by previous experience with conventional laparoscopic instruments in operation room or simulation area. Before the study, all the participants provided information about their previous experience, including on the simulation area. The protocol followed during the simulation task was explained to each participant before the task: 1. Maintain the rest position; 2. Insert the laparoscopic instrument; 3. Follow the circuit; 4. Reach the red 
dots and maintain the position during 3 seconds; 5 . Extract the laparoscopic instrument; 6. Maintain the rest position.

To perform a test that evaluate the effect produced by the postural freedom element. A prototype with just include this articulated element has been created and compared with a conventional fixed instrument with similar characteristics. Both instruments were tested using a test called "curve" (Fig.1). Curve is a test used by Matern et al. in 2004 [16] to compare laparoscopic instruments. On this test was included three points to acquire some static results. The participants had to follow the circuit without crossing the lines, these lines are spaced $20 \mathrm{~mm}$ from each other. A tip with ink was adapted to follow each trajectory.

Fig.1 Shape adapted by the "Curve" test used by Matern [16]. Three red dots included to acquire static positions.

To record the experiment, two cameras SMX - C100RP (Samsung, South Korea) were located one orthogonal to other. These cameras captured the front and the side of each participant. The distance between cameras and participants was 2 meters and the height was defined by the participant's height.

Tracker Video-Analysis Software (OPS, USA) was used to evaluate the camera records. This open software generates 25 results per second, tracking the elements selected on the scene. To provide a greater contrast the participants were dressed with white clothes, and two black markers were located in each joint (wrist, elbow and shoulder). This setup provided us results of angles and displacements of each joint during the experiment. Data analysis 


\section{was performed using the Kolmogorov-Smirnov and t-student tests in STATGRAPHICS Centurion XVI software.}

\section{Results}

The graphs below illustrate the movements of arm and forearm of the whole group of participants (Fig.2) and the movements performed by the experts (Fig.3) and novice participants (Fig.4).

Fig. 1 Shoulder and elbow movements. Control (red) and Prototype (blue). All the participants.

Fig. 2 Shoulder and elbow movements. Control (red) and Prototype (blue). Only expert participants.

Fig. 3 Shoulder and elbow movements. Control (red) and Prototype (blue). Only novice participants.

Table 1 presents the maximum shoulder and elbow displacement sort by previous experience.

Table 1 Maximum displacements presented with both instruments. Rows indicate the participants (all, experts and novices). Columns indicate the movements evaluated (shoulder adduction, shoulder abduction, shoulder flexion, shoulder extension, and elbow flexion).

\begin{tabular}{|c|c|c|c|c|c|c|}
\hline & & $\begin{array}{c}\text { Shoulder } \\
\text { Adduction }\end{array}$ & Shoulder & Shoulder & Shoulder & Elbow \\
abduction & flexion & extension & flexion \\
\hline \multirow{2}{*}{ Whole } & Control & 17,4 & 108,3 & 112,5 & 102,3 & 148,6 \\
\cline { 2 - 7 } & Prototype & 16 & 66,7 & 53,1 & 55,1 & 155,3 \\
\hline \multirow{2}{*}{ Experts } & Control & 5,5 & 108,3 & 84,4 & 53,8 & 143,2 \\
\cline { 2 - 7 } & Prototype & 16 & 66,7 & 53,1 & 55,1 & 143,9 \\
\hline \multirow{2}{*}{ Novices } & Control & 17,4 & 101,5 & 112,5 & 102,3 & 148,6 \\
\cline { 2 - 7 } & Prototype & 9,2 & 41,4 & 47,3 & 25,8 & 155,3 \\
\hline
\end{tabular}




\section{Discussion}

This study set out to assess the importance of the postural freedom in laparoscopic surgery. The positions adopted by the participants with both instruments presented differences, and the postural freedom concept reach better results in most of the movements evaluated.

The shoulder abduction and adduction values presented significant differences ( $\rho$ value $<00.5)$ between instruments. The prototype generated a range of movements of 14,77 degrees on this axis (Shoulder adduction=4,55; Shoulder abduction=10,21) and the control instrument presented a range of 25,89 degrees (Shoulder adduction=5,32; Shoulder abduction=20,56) (Error! Reference source not found.). The greater difference is presented in the abduction of the shoulder, this means that the participants performed lower arm displacements when is using the prototype. The reason is that the control instrument forces the participants to distance the arm from the neutral positions.

The shoulder flexion and extension results also showed differences. The prototype presented a range of movement of 20,12 degrees on this axis (Shoulder flexion=13,58; Shoulder extension=6,53) and the control instrument a range of 26,74 degrees (Shoulder flexion=18,58; Shoulder extension=8,15) (Error! Reference source not found.). Despite the difference presented on this axis looks no relevant, the maximum values obtained on these movements (Error! Reference source not found.) presented important differences. The 
movements traced by the prototype are more centered and the maximum displacements are half the size of the control instrument.

The prototype presents an elbow flexion deviation of 5,36 degrees lower than the control instrument (Prototype=6,59; Control=11,96). This result has been presented using the values of the Rapid Entire Body Assessment (REBA) [17]. The neutral position that REBA presents for this movement during a workrelated task is ranged between $60-100$ degrees of extension. The displacement generated by this two instruments are counted since the 80 degree. This means that both instruments maintain the participants in the neutral range.

The second question tackled during this study is the effect of the previous knowledge in the participant's adaptation. The results shown similarities in the movements traced by the expert participants with both instruments (Error! Reference source not found.). With the prototype, movements as shoulder abduction or shoulder flexion, presents greater benefits to the novice participants (Error! Reference source not found.). The values presented by the expert surgeons suggest that they improve their movements with the control instrument through the practice but these movements were emulated when they used the prototype. The novice participants presented higher values with the control instrument because they had not experience in the practice to improve their positions, but lower values with the prototype that means that the adaptation to this new concept was easier for participants without previous experience. 
The main goal of this study was to identify the effects of the articulated elements in MAS instruments, evaluating the Postural Freedom during MAS simulation in expert and novice participants.

The general results indicate that the concept of Postural Freedom provides positions closer to the neutral ones. Novice participants achieve better results in the test and higher differences between the control and the prototype instrument, this result indicate that the previous experience difficult the adaptation to new laparoscopic instruments and some training should be performed before the use during real practices.

The study supports the idea that the implementation of these kind of articulated elements could be the key to reduce the upper limb extreme displacements during laparoscopic practices. This improvement should be taken into consideration for developing future instrument designs.

Further motion analysis studies are required to verify this concept during long simulation trainings and real practices.

\section{REFERENCES}

1. Rau, A.C., Frecker, M.I., Mathew, A., Pauli, E.: Multifunctional Forceps for Use in Endoscopic Surgery-Initial Design, Prototype, and Testing. J. Med. Device. 5, 041001-1-041001-10 (2011). doi:10.1115/1.4005225

2. Awtar, S., Trutna, T.T., Nielsen, J.M., Abani, R., Geiger, J.: FlexDex TM: A Minimally Invasive Surgical Tool With Enhanced Dexterity and Intuitive Control. J. Med. Device. 4, 035003 (2010). doi:10.1115/1.4002234

3. Najmaldin, A., Guillou, P.: A Guide to Laparoscopic Surgery. (2000)

4. DiMartino, A., Done', K.N., Judkins, T.., Morse, J., Melander, J.: Ergonomic Laparoscopic Tool Handle Design. Hum. Factors. 673-677 (2004)

5. Papadoukakis, S., Kusche, D., Truss, M.C.: History of Laparoscopy, 
Endoscopic Extraperitoneal Radical Prostatectomy and Robotic Surgery. Endosc. Extraperitoneal Radic. Prostatectomy. 1-9 (2007)

6. Gao, H., Zhang, Z.: Laparoscopy Versus Laparotomy in the Treatment of High-Risk Endometrial Cancer: A Propensity Score Matching Analysis. Med. 94, e1245 (2015). doi:10.1097/md.0000000000001245

7. Berguer, R., Smith, W.D., Chung, Y.H.: Performing laparoscopic surgery is significantly more stressful for the surgeon than open surgery. Surg. Endosc. 15, 1204-1207 (2001)

8. Trejo, A., Jung, M.-C., Oleynikov, D., Hallbeck, M.S.: Effect of handle design and target location on insertion and aim with a laparoscopic surgical tool. Appl. Ergon. 38, 745-753 (2007)

9. Berguer, R., Gerber, S., Kilpatrick, G., Beckley, D.: An ergonomic comparison of in-line vs pistol-grip handle configuration in a laparoscopic grasper. Surg. Endosc. 12, 805-808 (1998). doi:10.1007/s004649900717

10. Berguer, R., Rab, G.T., Alarcon, A., Chung, J.: A comparison of surgeons ' posture during laparoscopic and open surgical procedures. Sport. Med. 139-142 (1997)

11. Nguyen, N.T., Ho, H.S., Smith, W.D., Philipps, C., Lewis, C., De Vera, R.M., Berguer, R.: An ergonomic evaluation of surgeons' axial skeletal and upper extremity movements (par during laparoscopic and open surgery. Ipar. Am J Surg. 182lpar, 720-4lpar (2001). doi:S0002961001008017 [pii]ppar

12. Trejo, A.E., Done', K.N., DiMartino, A.A., Oleynikov, D., Hallbeck, M.S.: Articulating vs. conventional laparoscopic grasping tools-surgeons' opinions. Int. J. Ind. Ergon. 25-35 (2006). doi:http://dx.doi.org/10.1016/j.ergon.2005.06.008

13. Herring, S.R., Trejo, A.E., Hallbeck, M.S.: Evaluation of four cursor control devices during a target acquisition task for laparoscopic tool control. Appl. Ergon. 41, 47-57 (2010)

14. Van Det, M.J., Meijerink, W.J.H.J., Hoff, C., Totté, E.R., Pierie, J.P.E.N.: Optimal ergonomics for laparoscopic surgery in minimally invasive surgery suites: A review and guidelines. Surg. Endosc. Other Interv. Tech. 23, 1279-1285 (2009). doi:10.1007/s00464-008-0148-x

15. Chandrasekaran, K., Thondiyath, A.: Design of a Two Degree-of-Freedom Compliant Tool Tip for a Handheld Powered Surgical Tool. J. Med. Device. 11, 014502 (2016). doi:10.1115/1.4034879

16. Matern, U., Kuttler, G., Giebmeyer, C., Waller, P., Faist, M.: Ergonomic aspects of five different types of laparoscopic instrument handles under dynamic conditions with respect to specific laparoscopic tasks: an electromyographic-based study. Surg. Endosc. 18, 1231-1241 (2004)

17. Hignett, S., McAtamney, L.: Rapid entire body assessment (REBA). Appl. Ergon. 31, 201-205 (2000) 
Click here to download Figure Fig. 1.jpg $\underline{\underline{\Perp}}$

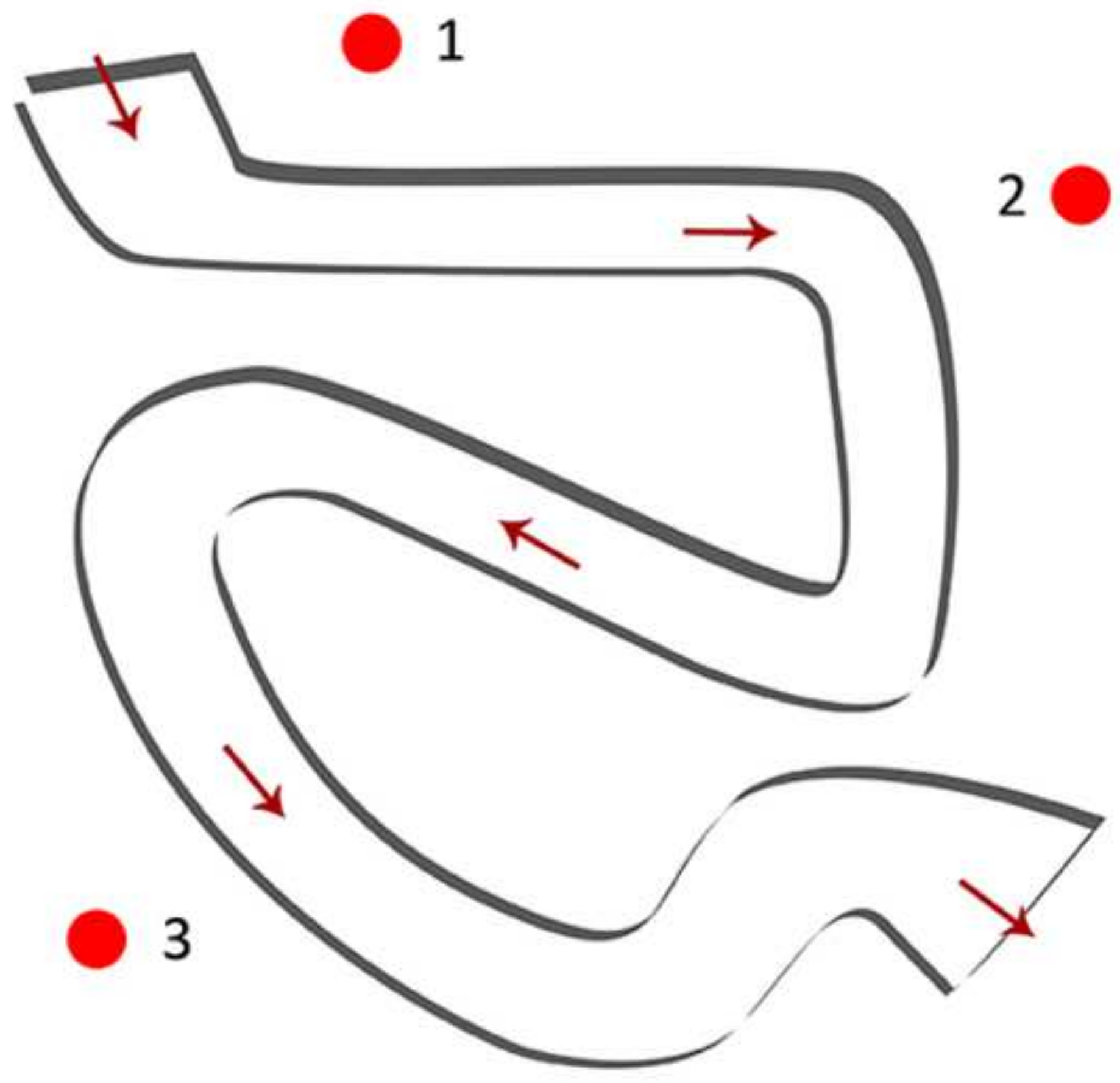




\section{All participants}

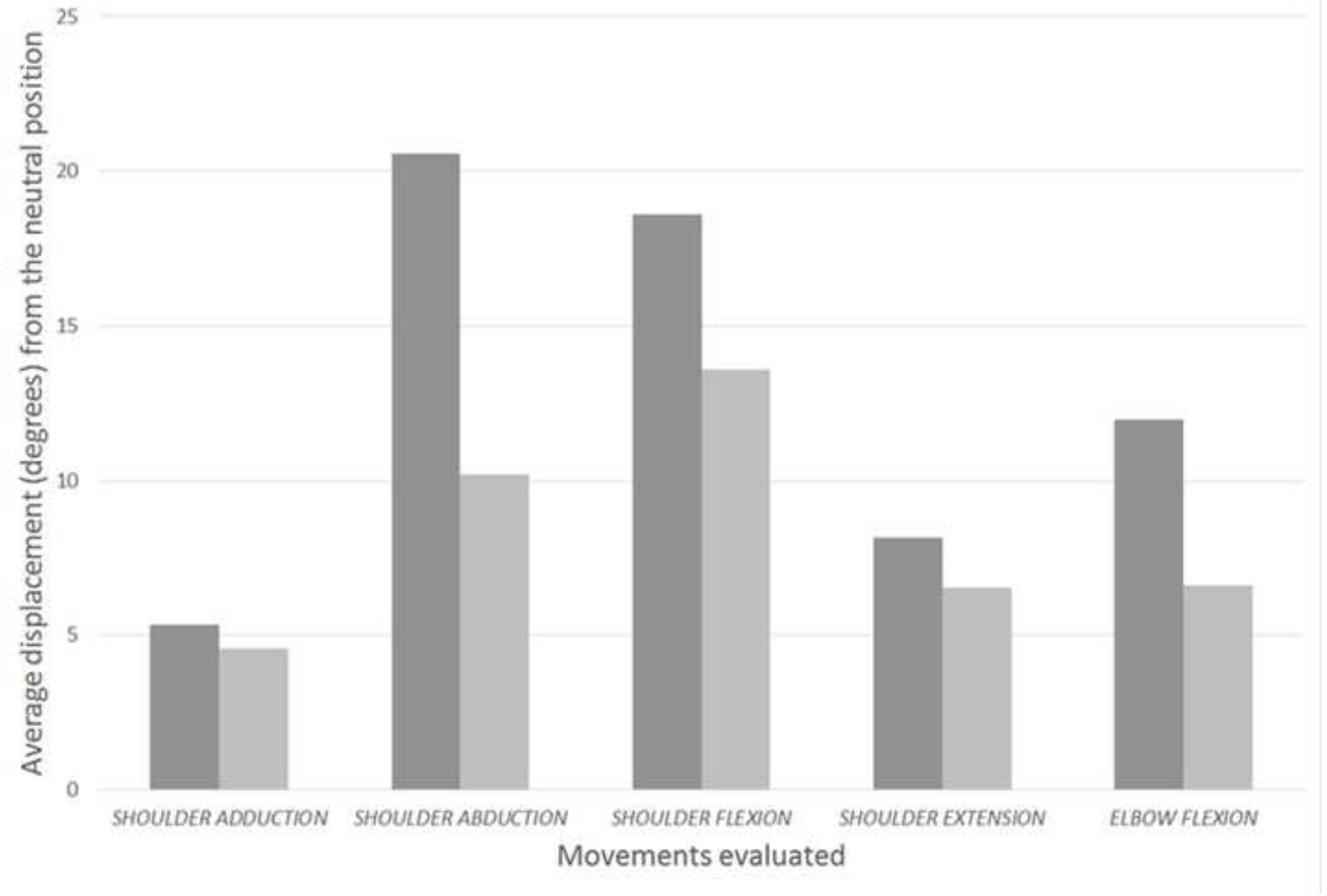




\section{Experts}

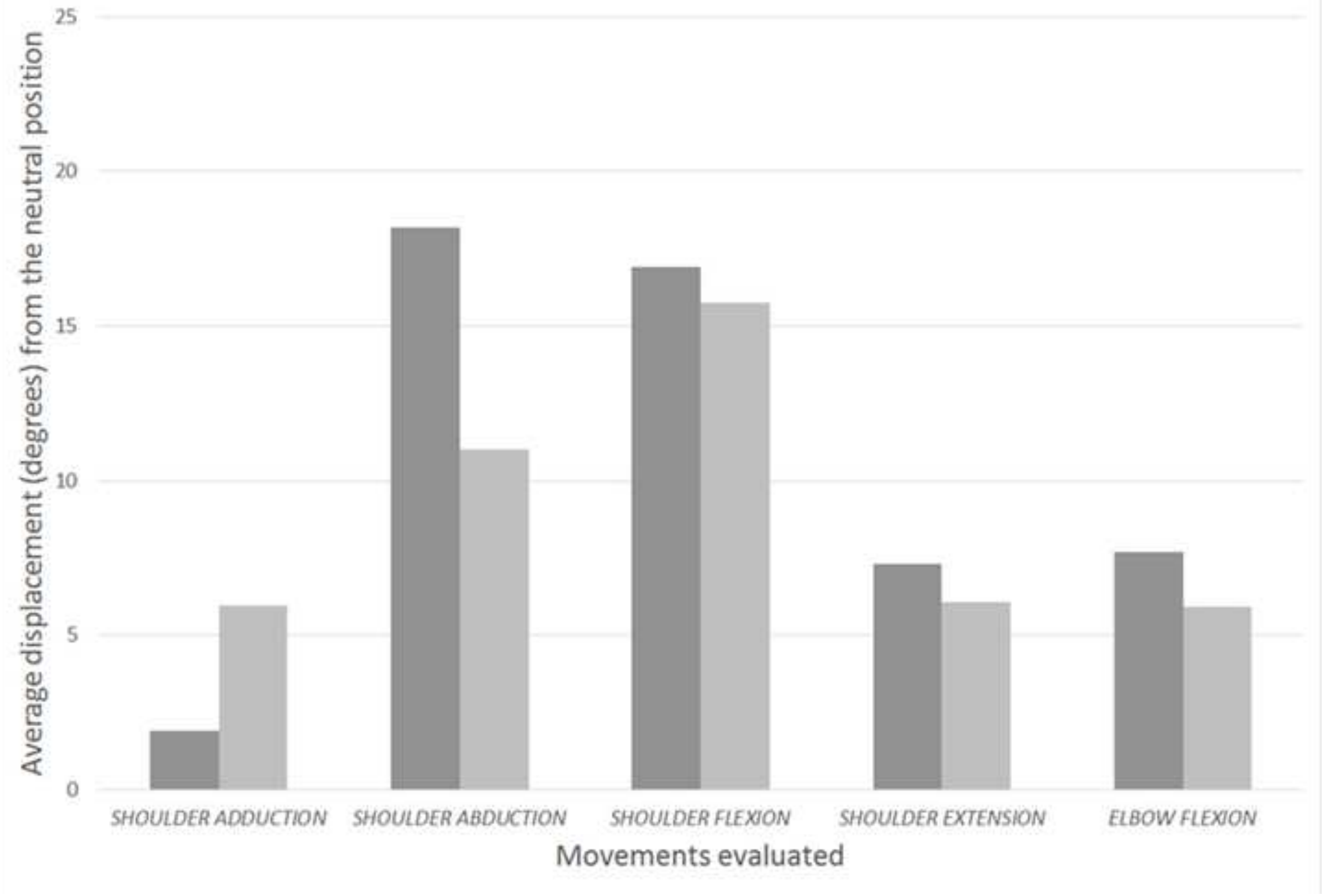




\section{Novices}

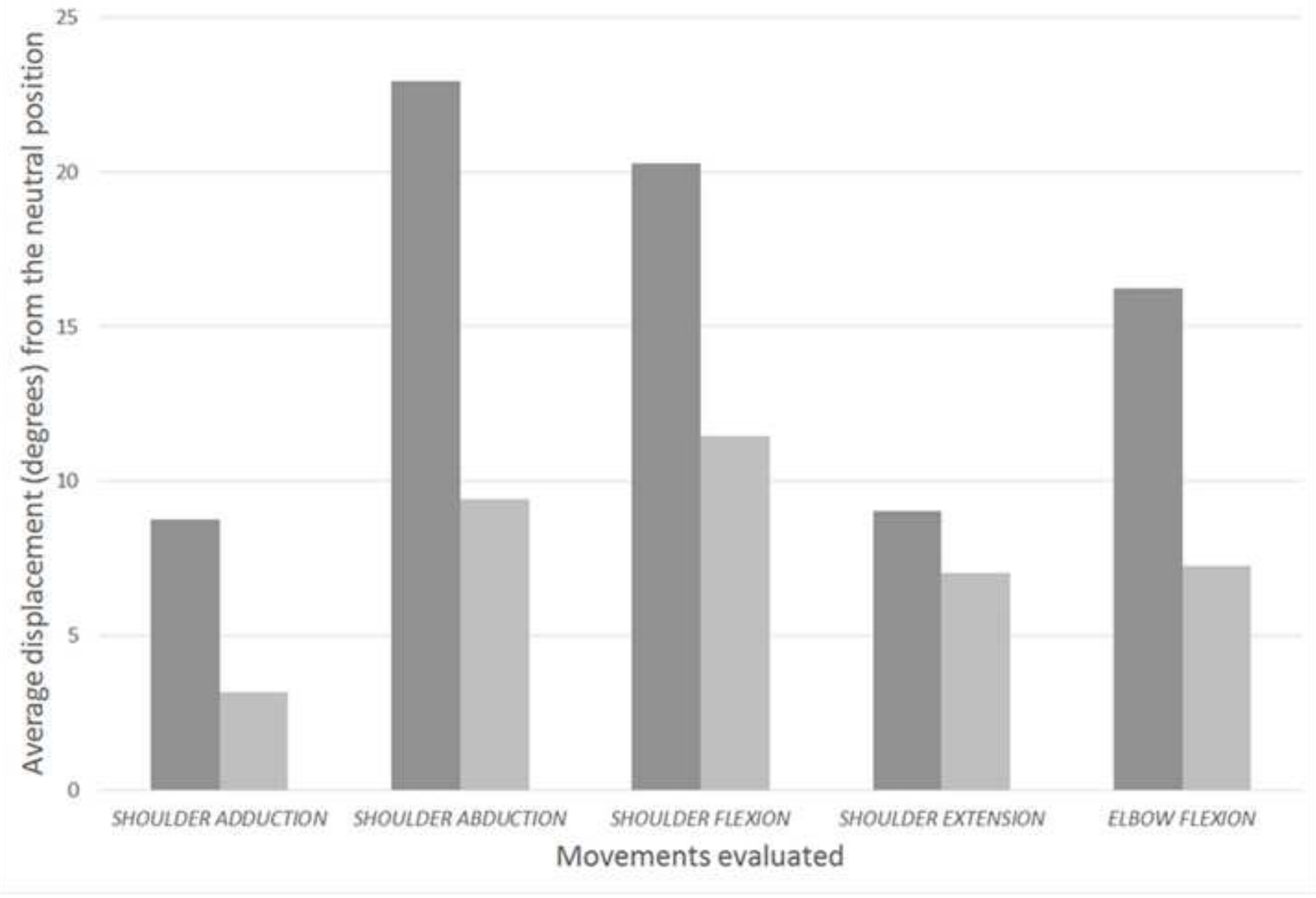

